\title{
Loss of the Surface Layers of Comet Nuclei
}

\author{
N. Thomas • C. Alexander • H.U. Keller
}

Received: 5 June 2007 / Accepted: 1 February 2008 / Published online: 26 February 2008

(C) Springer Science+Business Media B.V. 2008

\begin{abstract}
The Deep Impact observations of low thermal inertia for comet 9P/Tempel 1 are of profound importance for the observations to be made by the Rosetta spacecraft at comet 67P/Churyumov-Gerasimenko. While sub-surface sublimation is necessary to explain the observations, the depth at which this occurs is no more than $2-3 \mathrm{~cm}$ and possibly less. The low thermal conductivity when combined with local surface roughness (also observed with Deep Impact) implies that local variations in outgassing rates can be substantial. These variations are likely to be on scales smaller than the resolution limits of all experiments on the Rosetta orbiter. The observed physico-chemical inhomogeneity further suggests that the Rosetta lander will only provide a local snapshot of conditions in the nucleus layer.
\end{abstract}

Keywords Comets: surface $\cdot$ Comets: nuclei $\cdot$ Comets: emission

\section{Introduction}

High resolution observations of the surface of comet 9P/Tempel 1 returned by the Deep Impact mission (A'Hearn et al. 2005; Thomas et al. 2007; Belton et al. 2007) have added considerably to our knowledge of the surface layer of cometary nuclei. Although much remains to be learned, in conjunction with observations of Comet 1P/Halley (Giotto; Keller et al. 1996), Comet 19P/Borrelly (Deep Space 1; Soderblom et al. 2002) and Comet 81P/Wild 2 (Stardust; Brownlee et al. 2004), we can begin to place tighter constraints on the structure of the surface layer and the mass emission mechanisms from this layer. However, a feature of these new observations is that they indicate significant inhomogeneity.

\footnotetext{
N. Thomas $(\bowtie)$

Physikalisches Institut, Universität Bern, Sidlerstrasse 5, 3012 Bern, Switzerland

e-mail: nicolas.thomas@space.unibe.ch

C. Alexander

Jet Propulsion Laboratory, Pasadena, CA, USA

H.U. Keller

Max-Planck-Institut für Sonnensystemforschung, Katlenburg-Lindau, Germany
} 
While this was to be expected, the implications are that models require yet another degree of complexity and yet more parameters.

In this paper, we address the surface layer as a specific entity. We review the evidence for inhomogeneity and attempt to describe the forms (both physical and chemical) this inhomogeneity takes. We then discuss the surface heat balance and its implications for the mass emission mechanism. Here, we also discuss scale lengths and relate them to the resolutions of remote sensing instruments on the Rosetta orbiter. In doing so, we use a highly simplified thermal model to illustrate the importance of certain processes. In the following section, we describe our current concept of the structure of the surface layer and the relative distribution of volatile and non-volatile material in the uppermost few centimetres. Finally, we discuss how physical and chemical inhomogeneity naturally leads to further physical inhomogeneity in that the topography of the surface is affected by inhomogeneous physical and chemical properties. We conclude by reviewing possible strategies for the investigation of Comet 67P/Churyumov-Gerasimenko by the Rosetta spacecraft.

Throughout, we draw heavily on the recent book by Huebner et al. (2006) which provides an excellent review of the current status of thermal models of the surface layer.

\section{Evidence for Inhomogeneity}

Four comet nuclei have been investigated during close fly-bys by spacecraft in recent years. In interpreting these images, it is necessary to recall that the images were acquired by different imaging systems under different circumstances.

At first glance, it is clearly apparent that the surfaces of the nuclei exhibit different surface structures and features (Basilevsky and Keller 2006). 81P/Wild 2 is covered with circular and elliptical depressions. (Indeed this work was prompted by a challenge to explain these structures.) 19P/Borrelly, and, in particular, the central section of the observed nucleus, is relatively smooth with no obvious counterpart to the depressions seen on 81P/Wild 2. 9P/Tempel 1 is an intermediate. There are circular depressions but there are also large remarkably smooth areas. 1P/Halley, too, shows a circular depression and a large smooth region in the centre of the visible hemisphere. It is necessary to conclude therefore that topographically, the nuclei are different.

Furthermore, a single cometary nucleus can exhibit varied terrain. Britt et al. (2004) attempted to make a geomorphological map of 19P/Borrelly. They identified several regions on the nucleus with different surface properties. Smooth terrain dominated the central region of the nucleus while "mottled" terrain and dark spots were evident on the two ends of the elongated body. Nelson et al. (2004) attempted to determine whether these dark spots were albedo features or shadowed depressions. The central, smooth region appeared to show some vertical relief, which Britt et al. (2004) referred to as "mesas".

Deep Impact revealed remarkably varied terrain on the surface of 9P/Tempel 1. Smooth regions which may resemble the "mesas" in 19P/Borrelly images were clearly evident (although the surfaces of these features are relatively dark on 19P/Borrelly but relatively bright on 9P/Tempel 1). Rough surfaces were also seen. Variations in surface reflectance were prominent in several non-circular depressions. These were the only regions where water absorption features could be observed in infrared spectra of the nucleus (Sunshine et al. 2006). A central band of slightly lower reflectivity material could also be seen. From these observations, one must conclude that most nuclei are not physically homogeneous.

The spatial variation of activity on cometary nuclei also points to physical inhomogeneity. Dust emission from nuclei is not spatially uniform. The clearest example of this 
is 19P/Borrelly which, at the time of the DS1 fly-by in September 2001, exhibited a main jet roughly perpendicular to its long axis. This jet contributed 19-24\% of the total dust brightness (when compared to the whole at a fixed distance from the nucleus centre) and yet the full width at half maximum was only $18^{\circ}$ (Ho et al. 2007). The highest resolution images from DS1 show that this jet has three components at its base.

Several explanations of the origin of dust brightness enhancements in the inner coma of comets have been presented in the literature. Yelle et al. (2004) have suggested sub-surface emission through a vent (similar to a Laval nozzle) as a means of collimating the observed jet features. Keller et al. (1994) and Knollenberg (1994), following unpublished work by Kitamura in the 1980s (see also Kitamura 1986, 1987), investigated the effect of active areas surrounding an inactive central region. In this case, the gas, which drags the dust away from the nucleus, responds to the pressure gradient and "fills" the central region from all sides producing an enhancement in gas and dust density above the inactive surface. (In practise, the central region need not be completely inactive but merely of much lower activity than its surroundings.) Collimation of dust emission by concave topographic features has also been investigated by Knollenberg and has been shown to be a viable means of producing enhancements in dust brightness. Irrespective of the mechanism, whether it is topographic or a response to emission variations, the dust brightness variations seen in cometary comae indicate physical differences across the surface. It should be noted, however, that the gas, which drives the dust emission, cannot be seen with conventional imaging systems and Deep Impact observations of 9P/Tempel 1 suggest that the relationship between the spatial distribution of gas emission and that of dust emission is by no means trivial (M. A'Hearn et al., this issue).

The variations in surface reflectance may also point to chemical inhomogeneity. Although we know very little about the surface composition, there are measurable reflectance changes evident in the observations of 9P/Tempel 1. Reflectance variations might also be attributable to surface roughness and hence this is somewhat ambiguous. On the other hand, ground-based and spacecraft observations of jets in comets have been frequently made which show the jets to be rich (or poor) in $\mathrm{CN}, \mathrm{C}_{2}$, and $\mathrm{C}_{3}$ when compared to other jets from the same comet. Examples from diverse techniques include those given by Clairemidi et al. (1990), Schulz (1992), Henry et al. (2002), and Farnham et al. (2007). These radicals are minor species when compared to the total gas and dust production rate but they nonetheless indicate that one cannot assume that outgassing regions are chemically homogeneous.

From the above, it must be concluded that comet nuclei are neither uniform physically nor chemically. If we accept that comet nuclei are not merely different from each other but are also physically and chemically inhomogeneous in themselves, then it is apparent that surface features on a nucleus can be altered and modified as a result of this inhomogeneity. Hence, features on the surface are dynamic — the surface is not a uniformly shrinking iceball.

\section{Surface Heat Balance}

To address the importance of inhomogeneity for the surface layer in a more quantitative manner, we require a simple model to investigate numerous parameters. We begin by using the thermal conductivity equation in one-dimension for the internal heat transport, i.e.

$$
\rho c \frac{\delta T}{\delta t}=\kappa \frac{\delta^{2} T}{\delta x^{2}}
$$

where $\rho$ is the bulk density of the nucleus material, $c$ is its specific heat capacity, and $\kappa$ is the thermal conductivity such that the thermal diffusivity may be defined as $d_{t}=\kappa / \rho c$ and 
the thermal inertia, $\Gamma$, as $(\kappa \rho c)^{1 / 2}$. Here we ignore gas flow as a heat and mass transport mechanism within the nucleus and we also ignore effects such as amorphous-crystalline ice transition (which may or may not be a significant heat source) and radioactive decay heating. We keep open the possibility of explicitly describing conductivity changes within the nucleus. We do not attempt to describe further details of the heat transport as this would introduce further free parameters which cannot be constrained now (and indeed we have little hope of constraining them with Rosetta).

It is necessary to set boundary conditions. For the purposes of this study, we set explicitly the internal temperature of the nucleus. As this is still an unknown quantity, values in the range $30 \mathrm{~K}$ to $100 \mathrm{~K}$ can be easily justified. The surface boundary condition is the critical element in the study. The net energy flux excluding conduction is given by (Huebner et al. 2006)

$$
F_{\text {surf }}=\frac{S\left(1-A_{H}\right)}{r_{h}{ }^{2}} \cos \iota-\epsilon \sigma T^{4}-Q \Delta H
$$

where $\mathrm{S}$ is the solar flux at $1 \mathrm{AU}, r_{h}$ is the heliocentric distance [AU], $\iota$ is the zenith angle of the Sun at the surface under consideration ( $\cos \iota$ cannot of course be negative), $A_{H}$ is the hemispherical albedo ${ }^{1}, \epsilon$ is the IR emissivity (which is poorly known), $\sigma$ is the StefanBoltzmann constant, $Q$ is the mass loss rate resulting from sublimation and $\Delta H$ is the enthalpy of sublimation.

In principle, the system can now be solved using a discrete grid. However, Huebner et al. (2006) have shown the importance of being precise in specifying how the grid is treated. Here, for simplicity and ease of comparison, we use an explicit scheme and recognize that the Courant criterion

$$
\delta t \leq \frac{\rho c(\Delta r)^{2}}{2 \kappa}
$$

where $\delta t$ is the timestep, must be fulfilled. This results in timestep sizes of tens of seconds. Huebner et al. (2006) define the diurnal thermal skin depth, $l_{s}$, through the equation

$$
l_{s}=\sqrt{\frac{2 \kappa}{\omega \rho c}}
$$

where $\omega$ is the angular velocity of the nucleus $\operatorname{spin}\left(=2 \pi / \tau \mathrm{s}^{-1}\right.$ where $\tau$ is the spin period). By substituting values for compact ice and for what one might refer to as a highly porous material we arrive at the results in Table 1. It is generally appropriate to assume a grid size smaller than at least 1/4 of one skin depth (Spencer et al. 1989).

The Deep Impact spacecraft has given considerable support to the idea that the surface layers of comets are highly porous, low conductivity and therefore insulating. Groussin et al. (2007) report an estimated thermal inertia, $\Gamma$, of $<50 \mathrm{~W} \mathrm{~m}^{-2} \mathrm{~s}^{-1 / 2} \mathrm{~K}^{-1}$ (and therefore close to the values in the right hand column of Table 1). The thermal skin depth in this case must therefore be close to $2 \mathrm{~cm}-$ a quite remarkable result which has significant implications.

It will be immediately recognized that the thermal skin depth applies not merely in the vertical directions (into the interior) but also horizontally. This implies that, for example, a column into the interior will hardly be influenced by the thermal properties of a second column just a few centimetres away (at least on diurnal timescales).

\footnotetext{
${ }^{1}$ This is the ratio of the total photon power reflected to that incident. If one assumes a Lambertian surface, then $A_{H}=3 p / 2$ where $p$ is the geometric albedo.
} 
Table 1 Comparison of parameters of compact ice and highly porous material. Note the large difference in the thermal skin depth

\begin{tabular}{llll}
\hline Quantity & Compact ice & Units & Highly porous \\
\hline$\kappa$ & 1 & {$\left[\mathrm{~W} \mathrm{~m}^{-1} \mathrm{~K}^{-1}\right]$} & 0.01 \\
$\rho$ & 600 & {$\left[\mathrm{~kg} \mathrm{~m}^{-3}\right]$} & 400 \\
$c$ & 800 & {$\left[\mathrm{~J} \mathrm{~kg}^{-1} \mathrm{~K}^{-1}\right]$} & 400 \\
$\tau$ & 6 & {$[\mathrm{~h}]$} & 6 \\
$d_{t}$ & $2 \times 10^{-6}$ & {$\left[\mathrm{~m}^{2} \mathrm{~s}^{-1}\right]$} & $6.2 \times 10^{-8}$ \\
$\Gamma$ & 700 & {$\left[\mathrm{~W} \mathrm{~m} \mathrm{~s}^{-1 / 2} \mathrm{~K}^{-1}\right]$} & 40 \\
$l_{s}$ & 12 & {$[\mathrm{~cm}]$} & 2.1 \\
\hline
\end{tabular}

The Rosetta spacecraft is equipped with an orbiter and a lander. Considering the orbiter experiments first, the highest spatial resolution experiment onboard is the imaging system, OSIRIS (Keller et al. 2007). OSIRIS is foreseen to have a pixel scale of $1.86 \mathrm{~cm} \mathrm{px}^{-1}$ when $1 \mathrm{~km}$ above the surface (the expected closest distance of the spacecraft when preparing for the ejection of the lander) leading to an effective resolution of $\approx 4 \mathrm{~cm}$. For most normal operations, however, Rosetta may be several kilometres away and at periods of peak cometary activity it may be as much as 100-300 km from the nucleus. Hence, even under optimum conditions, OSIRIS will not resolve distances comparable to the thermal scale length.

While we have no evidence of inhomogeneity at the $10 \mathrm{~cm}$ scale, it is not unlikely based on observations so far. Furthermore, the highest resolution images we have from Deep Impact suggest that the surface is extremely rough in places (the "mesas" may be an exception but even this is not clear at $2 \mathrm{~cm}$ resolution). The low albedo of the surface would also suggest that this roughness continues down to sub-mm scales. The presence of the $\cos \iota$ term in the boundary condition implies that $2 \mathrm{~cm}$ scale roughness has a significant influence on the thermal balance. We must conclude that what we will see with Rosetta, even at the highest resolution, is an unresolved ensemble of discrete surface elements which barely influence each other and hence future thermal models must treat comet nucleus surfaces as such.

Naturally, the imaging system on the lander has spatially superior resolution. But it will only observe at one position on the nucleus and, given the expected inhomogeneity, these results will not be easy to extrapolate to other areas on the nucleus.

As discussed by Orosei et al. (1999) and re-iterated in Huebner et al. (2006), the treatment of the surface element in any numerical scheme of thermal diffusion can have significant influence on the results (particularly the predicted nighttime temperatures) if the thermal diffusivity is very low. A further problem arises, however, in that comet nucleus surfaces are unlikely to be smooth-more probably they are a rough accumulation of irregular particles. As has been pointed out by Davidsson (this issue), scattering in the uppermost layer will lead to absorption below the true surface. The importance of this will depend upon the particle properties of the uppermost layer. It is perfectly possible that significant energy deposition can occur up to several millimetres (several tenths of a scale length) below the surface. Hence, the use of a surface boundary for a grid element actually at the physical surface is also probably unrealistic.

We study a system with the properties shown in Table 2 to illustrate the importance of the boundary description.

In Fig. 1, we show the result of a simple calculation where we have integrated from aphelion to perihelion. For perihelion, we show the temperature with depth at the chosen position at midday and at midnight. Sublimation is excluded. The plot shows that the temperature drops with depth with a gradient of more than $3 \mathrm{~K} \mathrm{~mm}^{-1}$ at midday. While the code here has not been fully iterated to a steady state over the orbital period, it is also apparent 
Table 2 Parameters used for the evaluation of the examples shown herein
Orbit

Perihelion distance

$1.2923384 \mathrm{AU}$

Eccentricity

Rotation parameters

North pole vector wrt orbit normal

Rotation period

[0.5,0.,0.866]

$24 \mathrm{~h}$

Surface constants

IR emissivity

Hemispherical albedo

0.04

Thermal conductivity

$0.01 \mathrm{~W} \mathrm{~m}^{-1} \mathrm{~K}^{-1}$

Specific heat-density product

$2.410^{5} \mathrm{~J} \mathrm{~m}^{-3} \mathrm{~K}^{-1}$

Area under investigation

Longitude

$0.0^{\circ}$

Latitude

$+20.0^{\circ}$

Numerical quantities

Depth step size

$2.99 \mathrm{~mm}$

Timestep

$100 \mathrm{~s}$

Courant criterion

$107 \mathrm{~s}$

Lower boundary temperature

$90 \mathrm{~K}$

Depth of lower boundary

$5.98 \mathrm{~m}$

Orbital skin depth

$1.66 \mathrm{~m}$

Diurnal skin depth
Fig. 1 The temperature-depth profile at perihelion for the parameters shown in Table 2, excluding sublimation. Solid line: Midday. Dashed line: Midnight

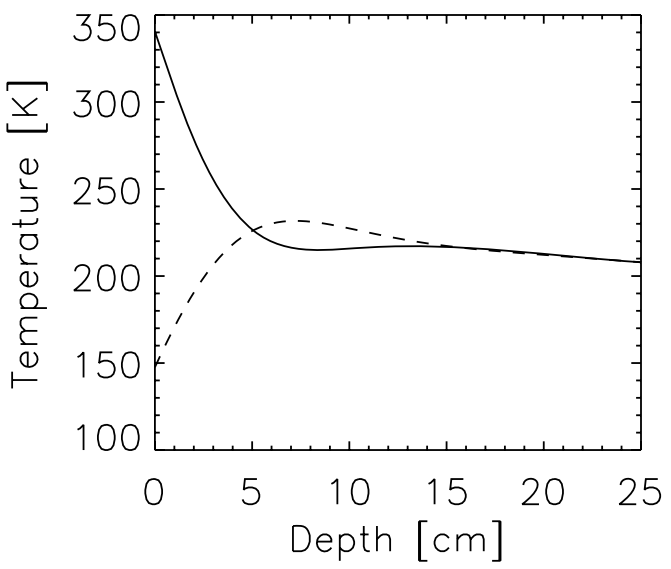

that the internal temperatures are below about $230 \mathrm{~K}$ only $5 \mathrm{~cm}$ below the surface and we note that we have used a relatively high temperature for the lower boundary condition.

The small thermal skin depth has major implications for theories of sub-surface sublimation. Several authors have suggested that sublimation occurs from below the visible surface. The Deep Impact observations by Sunshine et al. (2006) clearly established that the areal coverage of water ice on the surface of 9P/Tempel 1 was insufficient to explain the total water gas production rate of the comet and hence outgassing had to be occurring from below 
Fig. 2 The production rate of water gas computed for various depths of a low thermal conductivity surface layer. As the layer becomes thicker, the effective production rate per unit area decreases rapidly.

Calculation parameters are those shown in Table 2

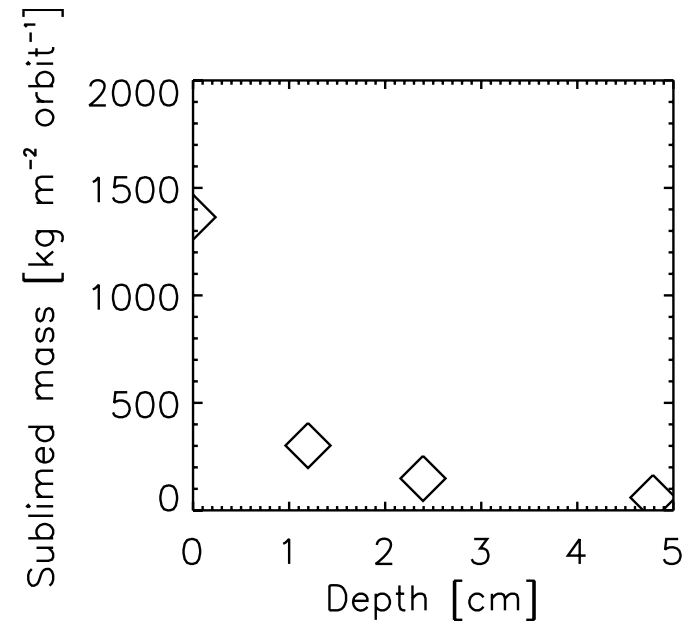

the observed surface in most regions. However, to be compatible with the thermal inertia observations and the implied thermal skin depth, this outgassing cannot be from depths greater than a couple of centimetres.

In Fig. 2, we show the total water gas production over one orbit plotted against the depth from which the emission occurs. We use the parameters given in Table 2. It should be noted that the inclusion of sublimation leads to numerical instability which in an explicit scheme must be compensated for by reducing the time step to well below (more than a factor of 10 below) the Courant criterion.

If the emission is at the surface, around $3 \mathrm{~m}$ of material is eroded per orbit which is equivalent to approximately 100 thermal skin depths. At peak production, one thermal skin depth would be eroded in no more than 2 nucleus rotations. Near-surface outgassing is therefore a highly dynamic process which can be strongly influenced on timescales of days by local inhomogeneities with depth.

The production rate per square metre drops rapidly and is reduced to only $4.4 \%$ of the production rate for a surface emission model just $4.8 \mathrm{~cm}$ below the surface. Let us take Comet 67P/Churyumov-Gerasimenko here as an example. The water gas production rate is around $10^{28}$ molecule $\mathrm{s}^{-1}$ at perihelion from an object with an average radius of $1.98 \mathrm{~km}$. Assuming free sublimation around $3 \%$ of the surface must be active to match the observed production rate. If we now demand sublimation from more than $2.5 \mathrm{~cm}$ below the surface, we reach a point where the whole illuminated nucleus must be uniformly active to match the production rate. As discussed above, Deep Impact, Deep Space 1, and Giotto have all shown that this is, even in the most optimistic interpretation, questionable for comets observed to date. Hence, we have a strict limit for the depth of any sub-surface outgassing.

\section{The Surface Layer}

Sublimation is occurring from a layer within $2.5 \mathrm{~cm}$ of the surface. This emitting layer is masked from view by an optically thick layer of non-volatile or considerably less volatile material (which we refer to, for brevity, as non-volatile material or NVM). The free sublimation rate of water ice at perihelion suggests erosion at a rate comparable to one skin depth in about 1 day. If emission is sub-surface then this rate might be reduced by up to a factor of 10 
Fig. 3 The surface temperature over one rotation at perihelion for two cases. Sublimation is modelled to occur below the surface at constant depth. Solid line: Sublimation occurring 1.2 $\mathrm{cm}$ below the surface. Dashed line: $4.8 \mathrm{~cm}$ below the surface

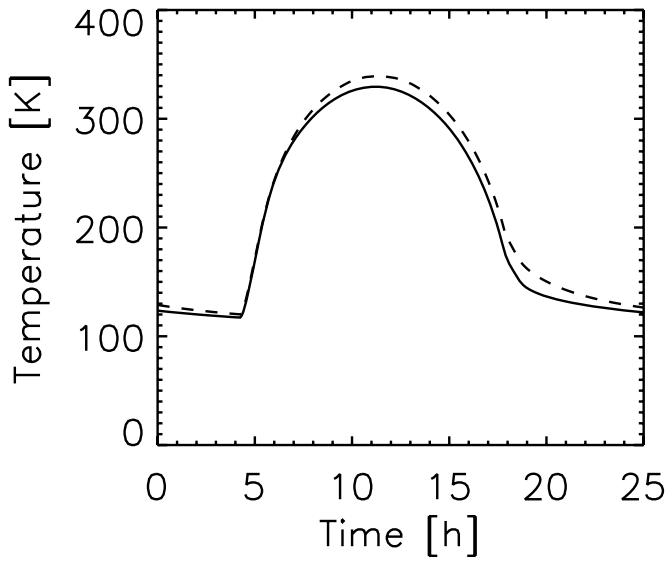

but no more, otherwise emission ceases as insufficient heat can be transported to maintain the outgassing rate necessary to match observed production rates. The NVM must therefore be removed on timescales of 10 days or less. Hence, the surface structure of the nucleus is continuously being modified even where NVM is present at the surface-it is dynamic.

Although sublimating ice is within $2.5 \mathrm{~cm}$ of the surface, it is mostly invisible to optical and infrared remote sensing. While Sunshine et al. (2006) made a detection of surface water ice, the areal coverage was completely insufficient to explain the observed gas production rate. This might be explained if the subliming material is at a depth of the order of a few hundred microns. Such a layer would be thin enough to support significant heat transport while masking, at optical and IR wavelengths, the ice below. The subliming material is then able to exert maximum pressure on the uppermost layer of crust or dust particles leading to their ejection. Gas sublimed actually at the surface does not collide with NVM and hence exerts little or no force on it.

If sublimation were to occur from $2 \mathrm{~cm}$ depth, for example, the sublimation, already reduced by the reduction in heat input, would have to be sufficient to disrupt the $2 \mathrm{~cm}$ thick layer above. (It is not reasonable to assume that deep sub-surface sublimation can pass through a $2 \mathrm{~cm}$ thick layer and then selectively erode a micron thick layer at the surface. If the surface were sufficient loose to be eroded by such a low gas flow rate, it should have been eroded earlier when the sublimation rate per unit area was higher.) It seems to be possible that such a process can occur. Observations of "chunks" of emitting material have been made on many occasions (e.g., Rodionov et al. 1998). However, this leads to ice being at the surface immediately after the ejection and may be a mechanism for small outbursts. It may also indicate inhomogeneity in the tensile strength of NVM with depth. The reproducibility of cometary emission over many rotations (e.g., Millis and Schleicher 1986) and the stability of total dust emission over many hours (Keller et al. 1996) suggests that such outbursts are not the "common" means of mass ejection (although it is not clear whether this is the dominant mass loss mechanism or not).

It is important to recognize that the depth of sub-surface sublimation will be extremely difficult to deduce from the observed surface temperature. In Fig. 3 we show the temperature of the surface in two cases. The solid line and the dashed line show the surface temperatures over one rotation when sublimation is occurring $1.2 \mathrm{~cm}$ and $4.8 \mathrm{~cm}$ below the surface respectively. The difference, under perfect model conditions, is typically only $5 \mathrm{~K}$. 


\section{Effects of Inhomogeneity}

\subsection{Surface Physical and Chemical Inhomogeneity}

The inhomogeneity of the comet nucleus, clearly evident in Deep Impact data, now allows us to build model structures and modify the outgassing properties of these structures in an infinite number of ways since the constraints are almost non-existent. The importance of exact knowledge of the surface properties is illustrated by increasing the thermal conductivity by a factor of 4 (from 0.01 to $0.04 \mathrm{~W} \mathrm{~m}^{-1} \mathrm{~K}^{-1}$ ) which corresponds to a doubling of the thermal inertia. If emission were to be from a depth of $2.5 \mathrm{~cm}$, the production over one orbit would increase by a factor of about 3.1.

The peak emission rate with the higher conductivity is $0.675 \mathrm{~g} \mathrm{~m}^{-2} \mathrm{~s}^{-1}$ and is around a factor of 3.4 higher than obtained with the lower conductivity. Hence, two adjacent regions with such a difference in thermal conductivity would produce an elevation difference of $2.9 \mathrm{~mm}$ in just one hour. The small-scale variations in conductivity of the surface layer would rapidly become evident in the local topography. There are additional non-linear feedbacks (both positive and negative) resulting from the production of surface slopes through such an inhomogeneity.

\subsection{Conductivity Changes with Depth}

Given the observed surface inhomogeneity, a change in thermal conductivity with depth is clearly possible. A simple model can be used to assess what influence this has on the surface temperature. In Fig. 4, the surface thermal conductivity has been set to $0.01 \mathrm{~W} \mathrm{~m}^{-1} \mathrm{~K}^{-1}$. At a depth of $1.3 \mathrm{~cm}$, the conductivity is abruptly changed to $0.04 \mathrm{~W} \mathrm{~m}^{-1} \mathrm{~K}^{-1}$. A comparison with a constant $\kappa$ of $0.01 \mathrm{~W} \mathrm{~m}^{-1} \mathrm{~K}^{-1}$ gives the following results. At perihelion, the midday temperature is $1.6 \mathrm{~K}$ higher if $\kappa$ is higher internally. The midnight temperature is, however, nearly $15 \mathrm{~K}$ lower. The reason for this is that, with the higher $\kappa$, the surface is more closely connected to the internal temperature (which has been set at $90 \mathrm{~K}$ ). Hence, investigations of the heat balance require accurate nighttime measurements of the surface temperature to place constraints not merely on the internal temperature but also the conductivity variation with depth.

Fig. 4 The depth dependence of temperature at midday and midnight at perihelion assuming a factor of 4 increase in thermal conductivity $1.3 \mathrm{~cm}$ under the surface. The dashed line shows the conductivity using the right-hand scale

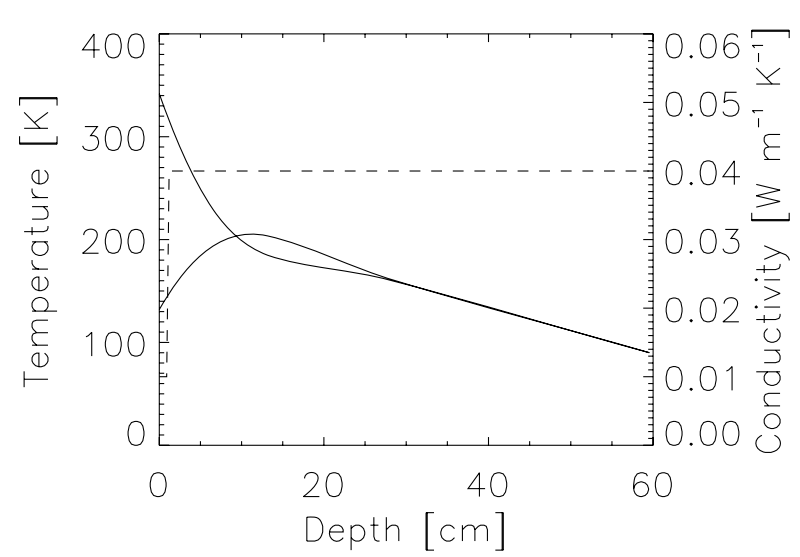




\subsection{Topographic Inhomogeneity}

We have already seen that the influence through thermal conduction of one nucleus element on another more than about $10 \mathrm{~cm}$ away is almost negligible because of the small thermal skin depth. This implies that as far as direct solar input is concerned, surface elements can be treated independently. This leads to independent development of that topography with time. For an initially spherical nucleus with zero obliquity, a homogeneous nucleus will sublime predominantly at equatorial latitudes to produce a "waist" (e.g., Dziak-Jankowska et al. 2002). (This situation is of course unstable to a change in the rotation axis so that the comet spins about the axis of minimum moment of inertia.) However, here we look at local effects.

Consider the geometry in Fig. 5. If the surface structure were located at the equator of a comet with zero obliquity, it is trivial to conclude that, integrated over one orbital period, the central, flat section of the structure will lose more mass than the surrounding inclined planes. This would result in the deepening of the pit until self-shadowing becomes significant. This picture rapidly becomes more complicated for moderate to high obliquities when combined with the eccentricity and the effective latitude of the active region. A relatively simple example is shown in Fig. 6. Here, we plot the cumulative sublimation over one orbit for the five surfaces in Fig. 5-a flat surface and four $45^{\circ}$ slopes arranged orthogonally to each other surrounding the flat surface. The direction to the nucleus north pole is also marked in the figure. (We note that Kossacki et al. (2003) have investigated a similar geometry applied to Mars.) It can be seen that the flat surface sublimes the most and hence the model structure should deepen as expected. The two slopes parallel in latitude (east and west facing slopes) behave in a rather similar way but lose $13 \%$ less mass. The slopes that are parallel to lines of longitude (north and south facing) show markedly different behaviour as one might expect because they strongly depend upon the latitude and the orientation of the rotation axis. In this case, the loss rates are lower and hence the pit expands asymmetrically. Over one orbit the expansion is $25 \%$ slower in the north-south direction than east-west.

This shows that, for example, an originally circular depression can then become elliptical on relatively short timescales. While this is not a demonstration of a production mechanism for the structures on 81P/Wild 2, it certainly shows that, given the number of free parameters available, an interpretation in terms of sublimation pits is feasible. It should also be noted that the rate of expansion differs through the orbit with the evolution of the southern slope occurring more slowly than the northern slope. Furthermore, the present example is rather

Fig. 5 A model geometry to demonstrate the effects of topography. The black central section is depressed but flat. The sides are at an angle of $45^{\circ}$ with respect to the local zero gradient

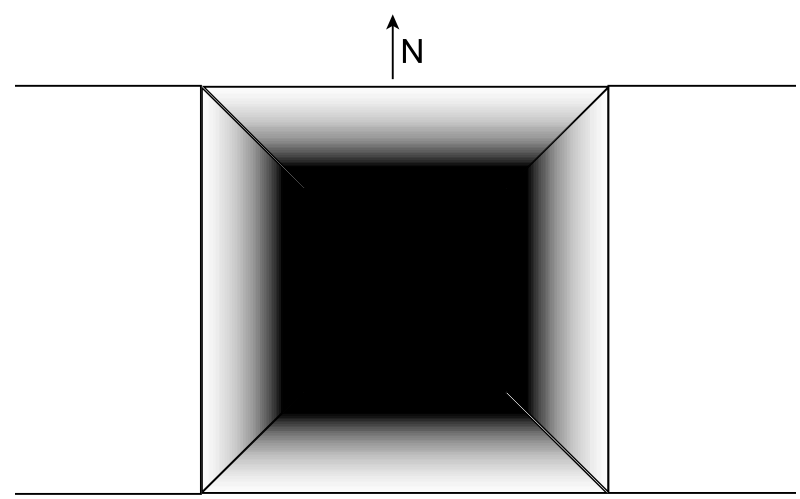


Fig. 6 Modelled cumulative sublimation of five surfaces on a comet with the orbital parameters given in Table 2. Solid: A flat surface. Other lines are for surfaces inclined at $45^{\circ}$.

Dash-dot-dot and short dashed lines are for surfaces with slopes aligned to lines of longitude. Dash-dot and long dashed lines are for surface with slopes aligned to line of latitude

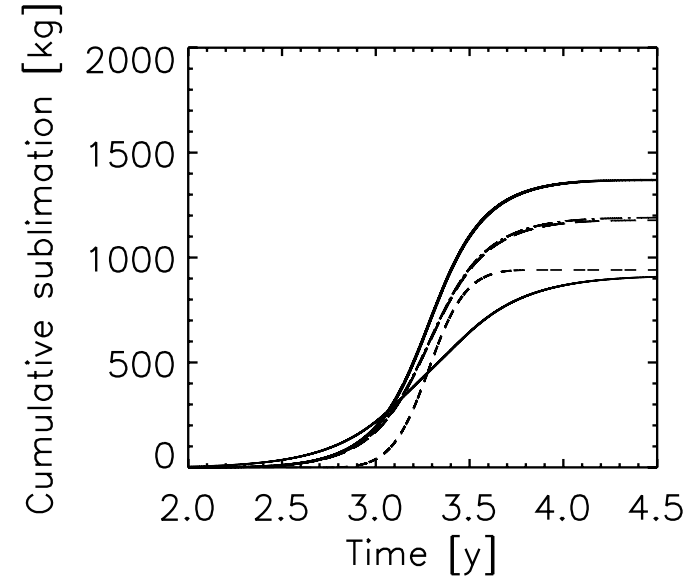

simple. Models with moderate obliquity and intermediate latitude sources can show more extreme behaviour. Investigation of these is on-going.

In this discussion, we have ignored "self-heating" of the nucleus. Topography will result in light being reflected from one surface element onto another. It is probably possible to ignore this because of the low hemispherical albedo of the nucleus. However, the nucleus surface can rise to temperatures close to $400 \mathrm{~K}$ when the comet is at $1 \mathrm{AU}$. This produces significant thermal IR emission which can add to the solar heat input of other surfaces within the emitting hemisphere. Ivanova and Shulman $(2002,2006)$ have studied this problem as have Kossacki et al. (2006) while Russell and Hecht (pers. comm.) have incorporated this physics into models of sublimation in mid-latitude Martian craters. What is not known here is the emission angular distribution function (which may be but is probably not isotropic because of sub-millimetre sized roughness) and hence more uncertainties remain.

\section{Strategies for Rosetta}

The implications for Rosetta of the Deep Impact results are quite profound.

Firstly, the lander carries several experiments to probe the interior of the nucleus. To constrain the sublimation process, however, penetration and measurement at very shallow depths is primarily required. Naturally, determination of the low boundary temperature and the interior structure support these observations but the dynamic nature of nucleus activity is governed by the upper $3 \mathrm{~cm}$.

Secondly, given the probable inhomogeneity of the nucleus, efforts must be made to place the lander in a representative region on the nucleus. This places a strong requirement on data acquisition and particularly analysis prior to lander site selection.

Thirdly, the importance of the microwave spectrometer, MIRO, for investigations of the surface layer have increased considerably. We can assume that the infrared spectrometer, VIRTIS, will fail to detect the source of the sublimation directly over most of the surface. It may also fail to place significant constraints on the depth of sublimation (within the first $3 \mathrm{~cm}$ ) via the temperature and thermal inertia. MIRO penetrates the surface to approximately this depth and can probe the temperature. While interpretation is unlikely to be straightforward it may be the only means of probing this critical region over most of the nucleus. It is necessary to get the spacecraft as close as possible to the nucleus to minimize the scale of 
the measurement. (The angular resolution of MIRO is equivalent to a spatial resolution of $5 \mathrm{~m}$ from a distance of $2 \mathrm{~km}$.)

Fourthly, the OSIRIS imaging system must be used to observe the nucleus at $<10 \mathrm{~cm}$ resolution near perihelion. This implies sending the spacecraft close to the nucleus while significant activity is on-going. The difficulties and dangers of this manoeuvre have not, to our understanding, been assessed in a complete way.

Finally, future modelling studies must take account of the fact that a 1-D representation of nucleus activity ignores unresolved roughness and inhomogeneity which will play a critical role in determining the local gas and dust emission which in turn will influence the nearnucleus coma structure.

\section{Conclusions and Discussion}

The Deep Impact observations have added considerably to our understanding of the surface layer of cometary nuclei. Observations of surface inhomogeneity, both topographical and chemical, have been made (A'Hearn et al. 2005; Belton et al. 2007) and the thermal inertia of the uppermost layer is extremely low (Groussin et al. 2007). Sub-surface chemical heterogeneity cannot be ruled out. This leads to a series of important consequences.

The thermal skin depth is of the order of $2 \mathrm{~cm}$. But, sub-surface sublimation must be occurring to explain the limited surface area coverage of water ice seen by Deep Impact (Sunshine et al. 2006). The depth below the surface of sublimation is therefore limited to a few centimetres at most. The surface layer is highly dynamic with disruption of a thickness equivalent to one thermal skin depth in less than one day occurring at perihelion for typical Jupiter-family nuclei. Inhomogeneity on scalelengths comparable to the thermal skin depth results in modifications to local topography which can significantly affect the local heat balance on similar timescales. The resolution of the Rosetta imaging system, OSIRIS, will typically be five times greater than the thermal skin depth. Hence, inhomogeneities on the scale of the skin depth will be observed as an ensemble of different emission properties which must be modelled as such. One-D calculations (or multi-dimensional calculations using surface grid sizes much larger than the thermal skin depth) will fail to give an accurate picture of the parameters providing the emission unless some approach to compensating for the effects of inhomogeneity is taken.

The temperature variation with depth can be probed best by MIRO and this is likely to provide the most significant constraints on the thermo-physical properties of the surface layer. Variations in thermal conductivity with depth have a limited effect on the surface daytime temperature but might be addressed by measuring the thermal IR from the nightside if VIRTIS is capable of this task.

Models clearly show that surface structures similar to some of those observed on comet nuclei can be produced by sublimation processes together with topographic effects. The observed inhomogeneity in comets gives an enormous number of additional free parameters for thermo-physical modelling. This implies that, at the present time, our knowledge is so limited that essentially any realistic structure can be produced by using the correct mix of input parameters. Progress in this field will be somewhat arbitrary until further constraints become available.

Acknowledgements The authors thank the organizers, both referees for accurate comments, and in particular Elmar Jessberger for asking a provocative question! 


\section{References}

M.F. A'Hearn et al., Science 310, 258 (2005)

A.T. Basilevsky, H.U. Keller, Planet. Space Sci. 54, 808 (2006)

M.J.S. Belton et al., Icarus 187, 332 (2007)

D.T. Britt et al., Icarus 167, 45 (2004)

D.E. Brownlee et al., Science 304, 1764 (2004)

J. Clairemidi, G. Moreels, V.A. Krasnopolsky, Icarus 86, 115 (1990)

B. Dziak-Jankowska, J. Leliwa-Kopystyński, M.E. Królikowska, Earth, Moon, Planets 90, 35 (2002)

T.L. Farnham, N.H. Samarasinha, B.E.A. Mueller, M.M. Knight, Astron. J. 133, 2001 (2007)

O. Groussin et al., Icarus 187, 16 (2007)

F. Henry, D. Bockelée-Morvan, J. Crovisier, J. Wink, Earth, Moon, Planets 90, 57 (2002)

T.-M. Ho, N. Thomas, D.C. Boice, M. Combi, L.A. Soderblom, V. Tenishev, Planet. Space Sci. 55, 974 (2007)

W.F. Huebner, J. Benkhoff, M.T. Capria, A. Coradini, C. De Sanctis, R. Orosei, D. Prialnik, Heat and gas diffusion in comet nuclei. ISSI Scientific Report, SR-004, 2006, ISBN 1608-280X

A. Ivanova, L. Shulman, Earth, Moon, Planets 90, 249 (2002)

A.V. Ivanova, L.M. Shulman, Adv. Space Res. 38, 1932 (2006)

H.U. Keller et al., Space Sci. Rev. 26 (2007)

H.U. Keller, J. Knollenberg, W.J. Markiewicz, Planet. Space Sci. 42, 367 (1994)

H.U. Keller, W. Curdt, J.R. Kramm, N. Thomas, Images of the Nucleus of Comet Halley. ESA-SP 1127, vol. 1,1996

Y. Kitamura, Icarus 66, 241 (1986)

Y. Kitamura, Icarus 72, 555 (1987)

J. Knollenberg, Ph.D. Thesis. Univ. Göttingen, 1994

K.J. Kossacki, W.J. Markiewicz, M.D. Smith, Planet. Space Sci. 51, 569 (2003)

K.J. Kossacki, J. Leliwa-Kopystyński, S. Szutowicz, Icarus 184, 221 (2006)

R.L. Millis, D.G. Schleicher, Nature 324, 646 (1986)

R.M. Nelson, L.A. Soderblom, B.W. Hapke, Icarus 167, 37 (2004)

R. Orosei, F. Capaccioni, M.T. Capria, A. Coradini, M.C.D. Sanctis, C. Federico, M. Salomone, J.-P. Huot, Planet. Space Sci. 47, 839 (1999)

A.V. Rodionov, L. Jorda, G.H. Jones, J.F. Crifo, F. Colas, J. Lecacheux, Icarus 136, 232 (1998)

R. Schulz, Icarus 96, 198 (1992)

L.A. Soderblom et al., Science 296, 1087 (2002)

J.R. Spencer, L.A. Lebofsky, M.V. Sykes, Icarus 78, 337 (1989)

J.M. Sunshine et al., Science 311, 1453 (2006)

P.C. Thomas et al., Icarus 187, 4 (2007)

R.V. Yelle, L.A. Soderblom, J.R. Jokipii, Icarus 167, 30 (2004) 